\title{
Um jogo sério para auxiliar o ensino-aprendizagem de estudantes de medicina
}

\author{
Jesaías Carvalho Pereira Silva, UFRN e UFERSA, jesayassilva@gmail.com \\ Everton Jales de Oliveira, UFERSA, verto.jales@gmail.com \\ Ademar França de Sousa Neto, UFCG, ademarneto@ufersa.edu.br \\ Leonardo Torres Marques, UFC, leonardo.torresmarques@gmail.com \\ Bruno de Sousa Monteiro, UFERSA, brunomonteiro@ufersa.edu.br \\ Araken de Medeiros Santos, UFERSA, araken@ufersa.edu.br \\ Francisco Milton Mendes Neto, UFERSA, miltonmendes@ufersa.edu.br
}

\begin{abstract}
Resumo: A rápida expansão da rede de faculdades de medicina trouxe um déficit na qualidade de ensino, devido à falta de infraestrutura e recursos em algumas universidades, prejudicando, assim, a formação teórica e prática dos alunos. Porém, com os avanços significativos das tecnologias móveis nos últimos anos, as instituições de ensino estão a proporcionar novos contextos e oportunidades de aprendizagem e ensino. Assim, os jogos sérios podem ser utilizados para auxiliar no ensino e aprendizagem dos estudantes. Com base neste contexto, foi desenvolvido um simulador/jogo sério com o objetivo de auxiliar o aprendizado dos alunos de medicina em casos clínicos e assuntos em gerais da área. $\mathrm{O}$ trabalho conta com dois projetos, o primeiro é o jogo sério mobile e o segundo é um algoritmo de aprendizado de máquina e múltiplos classificadores, utilizado para classificar doenças. Os resultados da avaliação mostram indícios de que a ferramenta tem potencial para servir de auxílio para o ensino aprendizagem para os potenciais usuários. Palavras-Chave: Jogo Sério. Simulador. Medicina. Aprendizagem. Aprendizado de Máquina.
\end{abstract}

\section{A serious game to aid the teaching-learning of medical students}

Abstract. The rapid expansion of the medical school network brought a deficit in the teaching quality due to a lack of infrastructure and resources in some universities, thus hampering students' theoretical and practical training. However, with significant advances in mobile technologies in recent years, educational institutions provide new contexts and learning and teaching opportunities. Thus, serious games can assist in the teaching and learning of medical students. Based on this context, we developed a serious game to help medical students learning in clinical cases and general subjects in the area. This paper describes two projects: a mobile serious game and a machine learning algorithm as multiple classifiers to classify diseases. Evaluation results show evidence that the tool can serve as a teaching aid for potential users.

Keywords: Serious Game. Simulator. Medicine. Learning. Machine Learning.

\section{Introdução}

Dados do Conselho Regional de Medicina do Estado de São Paulo (CREMESP) indicam que os estudantes de medicina têm obtido baixo desempenho no exame do CREMESP ao longo dos anos, o qual avalia conteúdos básicos de áreas consideradas essenciais na Medicina. Mais da metade dos estudantes de medicina, em 2016, foram reprovados nesse exame. Dos 2.677 participantes, 56,4\% foram reprovados. Os estudantes não acertaram 60\% da prova (CREMESP 2017). Em 2017, foi constatado que, dos 2.636 participantes, $60 \%$ demonstraram pouco conhecimento sobre doenças parasitárias, formas de veiculação e contaminação (CREMESP 2018). Estes dados são 
alarmantes, visto que os alunos, após o curso, lidarão com vidas humanas, havendo risco para a própria população. Enquanto isso, em 2018, os resultados foram melhores, mas ainda preocupam, pois dos 3.174 participantes, $38,2 \%$ foram reprovados no exame.

Diante desse quadro, fica visível uma má formação dos estudantes de medicina, que acarreta em erros médicos que afetam toda a sociedade. Esses erros têm sido uma das grandes causas de mortes no mundo. James (2013) realizou uma revisão da literatura que estimou danos evitáveis usando uma análise ponderada e descreveu uma faixa de incidência de 210.000 a 400.000 mortes, por ano, associada a erros médicos em pacientes hospitalares nos Estados Unidos, tomando como base estudos publicados de 2008 a 2011. No Brasil, a cada hora, seis pessoas sofrem de erro no diagnóstico ou negligência médica, havendo os erros clínicos uma relação com isso (Couto et al. 2018). A prática clínica é cheia de vícios, condutas e conceitos errados e, determinadas condutas, podem causar danos ao paciente e configura má prática clínica (Lopes 2020).

Portanto, é perceptível uma lacuna na formação dos estudantes de medicina. Essa lacuna pode ser suprida por meio de jogos sérios, pois vem ganhando grande força no ensino-aprendizagem por possibilitar a reprodução de situações do mundo real em ambientes criados em computador (Bogoni e Pinho 2014), sendo utilizados como métodos de aprendizado ativo para incentivar alunos a revisar materiais. Este tipo de aprendizagem possui boa aceitação por parte dos professores e alunos de medicina. (Castro e Siqueira 2017).

Com isso, o objetivo do artigo é apresentar o DocTraining mobile e sua validação. Neste jogo/simulador, os estudantes de medicina podem resolver casos clínicos, acessar salas virtuais de perguntas e atender pacientes virtuais oferecidos ao jogo por meio de um web service, que possui um sistema de aprendizado máquina (AM). A validação do jogo sério foi com os estudantes de medicina que tiveram a oportunidade de usá-lo, seguindo o Modelo de Aceitação de Tecnologia (TAM), proposto por Davis (1989).

O restante deste trabalho está estruturado da seguinte maneira: primeiro, na Seção 2, estão apresentados os trabalhos relacionados; na Seção 3, é detalhado o DocTraining Mobile, bem como o sistema para gerenciar os dados, o sistema com o aprendizado de máquina e o web service; a validação é apresentada na Seção 4; e, por fim, na Seção 5, são apresentadas as considerações finais, ameaças à validade e trabalhos futuros.

\section{Trabalhos Relacionados}

Nesta Seção estão apresentados três trabalhos relacionados à temática. O APS Game, desenvolvido por França et al. (2016), é um jogo educativo proposto para consolidar conceitos teóricos por meio da simulação de casos clínicos, sendo uma alternativa inovadora para a educação profissional em saúde no âmbito da atenção primária. $\mathrm{O}$ jogo consiste no atendimento de casos clínicos, passando pelas etapas de anamnese, exames físicos e exames complementares. Dessa forma, o jogo passa por todas as principais etapas de um atendimento clínico no âmbito da atenção primária ofertada na rede pública. O objetivo do jogador é realizar o diagnóstico do caso clínico e indicar o tratamento mais adequado. Após concluir todas as etapas, um feedback é dado ao jogador com base nas suas decisões durante a condução do caso, apontando eventuais equívocos, reforçando o que foi recomendado corretamente e apontando conteúdos e alternativas de acordo com cada situação.

O SimDeCS (Simulação para tomada de Decisão no Serviço de Saúde), apresentado por Flores et al. (2013), foi desenvolvido por especialistas das áreas da informática, educação e saúde, com o objetivo de realizar o monitoramento do usuário/estudante de medicina durante o processo de simulação, fornecendo feedback e orientação sobre as decisões clínicas tomadas. O simulador trabalha o desenvolvimento das capacidades 
técnicas e competências no diagnóstico formulado, seguindo o próprio ritmo de aprendizagem do usuário/jogador.

O Geriasims, desenvolvido pelos pesquisadores Orton e Mulhausen (2008), é um simulador de pacientes virtuais para a educação em geriatria. Ele consiste na criação de um grupo de pacientes virtuais a ser disponibilizado a estudantes de medicina e apresenta os princípios fundamentais e as práticas de avaliação e cuidados geriátricos.

Os trabalhos apresentados objetivam melhorar o ensino aprendizagem dos estudantes de medicina. Contudo, não foram encontrados trabalhos que, para classificar as doenças, utilizando os sintomas, use múltiplos classificadores (MC) de AM (Silva et al. 2019). O presente trabalho disponibiliza um sistema on-line de gerenciamento de dados, tornando a aplicação flexível, deixando o professor gerenciar as amostras de doenças que deseja trabalhar.Além disso, é apresentado um web service, com métodos de solicitações, que podem servir de padrão para outras aplicações que tenham o mesmo propósito.

\section{DocTraining Mobile}

O DocTraining Mobile foi dividido em dois projetos, A e B, como apresentado na Figura 1. O projeto A corresponde ao jogo sério para smartphones descrito neste trabalho com toda a estrutura necessária para interação do jogador com os casos clínicos e outros elementos de aprendizagem e jogabilidade, na qual os estudantes de medicina podem acessar via smartphones com acesso à internet. Os dados do jogo sério são provenientes de amostras de doenças reais, que são classificadas com o algoritmo de AM.

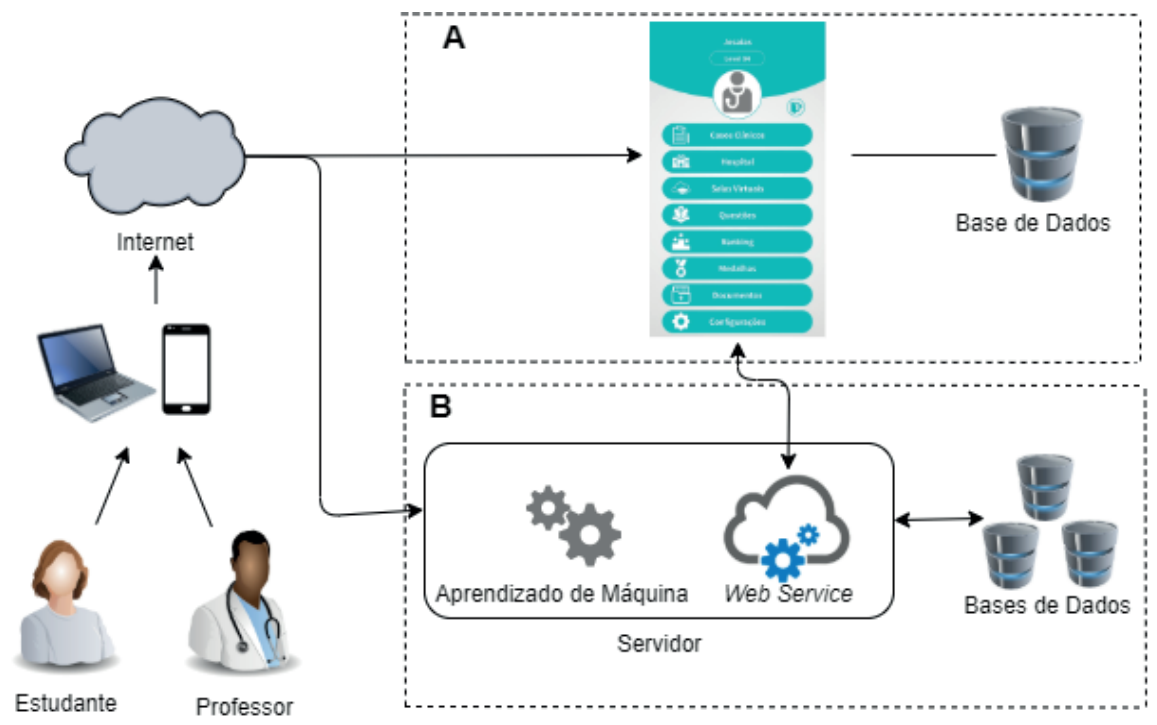

Figura 1 - Visão geral do DocTraining.

O projeto $\mathrm{B}$ corresponde à classificação de dados utilizando os algoritmos de $\mathrm{AM}$ e de MC. Esses projetos são integrados utilizando um web service, que fornece a integração dos dois projetos, sem considerar a plataforma de desenvolvimento ou o ambiente operacional, tornando-os independentes (Fu et al. 2015). Também há um web site para divulgação e gerenciamento do jogo.

O jogo mobile/simulador do DocTraining pode ser executado em ambientes de sistema operacional Android. Ao abrir o aplicativo é solicitado usuário e senha para que possa utilizar o aplicativo. Caso não tenha uma conta é possível criá-la. Dentro do aplicativo, o estudante tem as opções de atender pacientes virtuais no hospital, atender casos clínicos, entrar em salas virtuais, questões diversas, além de ranking, medalhas, documentos da área da medicina e configurações, este menu pode ser observado na Figura 2a. 


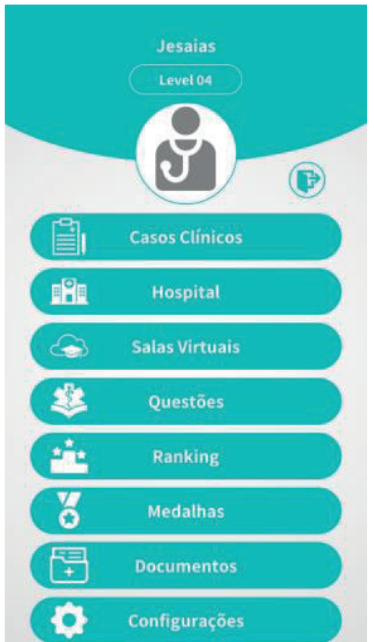

a) Menu Principal

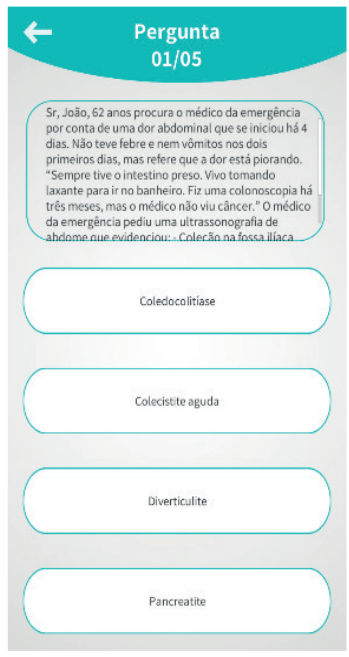

(b) Caso clínico

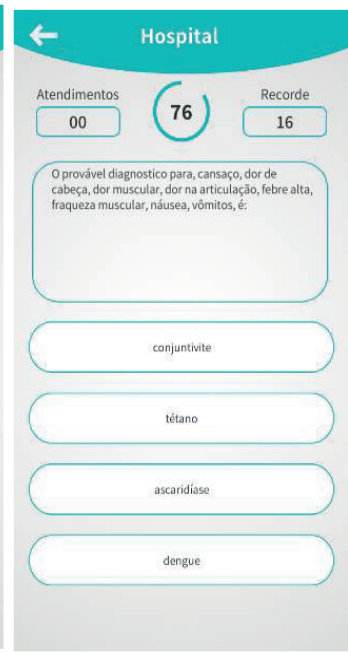

(c) Hospita

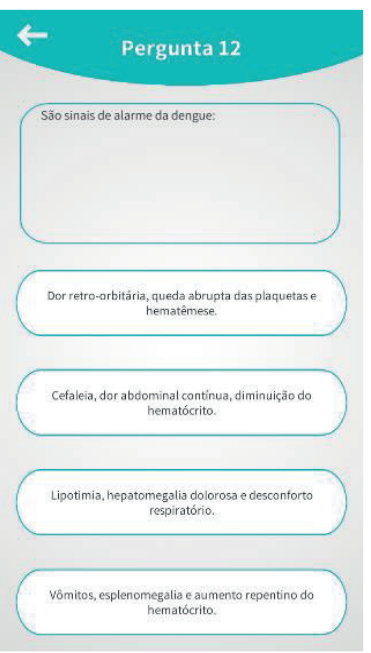

(d) Salas virtuais

Figura 2 - Telas do DocTraining Mobile e do Sistema On-line.

Os Casos clínicos contêm questões variadas, que são do dia a dia dos médicos. Ao atender esses casos clínicos, os estudantes de medicina adquirem conhecimento sobre esses problemas do cotidiano. Na Figura $2 \mathrm{~b}$ é apresentado um exemplo: o estudante possui Quatro alternativas e deve escolher uma. Esses casos clínicos são controlados por níveis/fases, na qual os estudantes podem avançar de nível e/ou resolver questões de níveis anteriores para rever e treinar novamente. O jogo atribui notas em cada nível, essas notas são indicadas por estrelas, que variam de uma até três.

Nas salas virtuais, é possível responder diversas questões específicas da medicina, divididas por salas, em que cada uma é de um assunto. Cada pergunta possui quatro alternativas, sendo apenas uma verdadeira que é previamente cadastrada, isso pode ser observado na Figura 2d. Esses dados são incrementados por um sistema on-line, onde os professores ou especialistas da área da medicina, com acesso ao sistema on-line, podem inserir novas salas e/ou novas perguntas, na Subseção 3.1 são apresentados mais detalhes do sistema on-line.

Dentro do hospital são apresentados pacientes virtuais que são controlados por um módulo inteligente com AM. Este módulo simula problemas de saúde utilizando amostras de sintomas cadastrados pelos profissionais de saúde. O estudante inicia no hospital médico com 80 segundos, o objetivo é verificar os sintomas que os pacientes apresentam e dar os diagnósticos corretos, isso pode ser observado na Figura 2c. Inicialmente, caso acerte, são adicionados 10 segundos ao tempo, caso erre são subtraídos quatro segundos. A partir de determinados atendimentos, a quantidade ganha, por acertar um diagnóstico, é diminuída, isso simula uma situação em que é essencial dar o diagnóstico correto em um menor tempo possível. Ao zerar o cronômetro é chegado ao fim do atendimento no hospital. O hospital, as salas, os casos clínicos e as questões diversas geram Pontos por Experiência (XP), para que os jogadores se sintam motivados nas opções de ranking a melhorar suas pontuações. Outras pontuações são de quantidade de atendimentos e de questões respondidas. A partir de determinado XP, atendimento ou respostas são desbloqueadas algumas conquistas, que são troféus para o estudante. $\mathrm{O}$ jogo sério foi desenvolvido na engine Unity $3 D$, utilizando a linguagem de programação $C \#$.

\subsection{Sistema On-line para Gerenciamento de Dados}


O projeto conta com um site $e^{l}$, podendo ser acessado por qualquer computador com acesso à internet. $\mathrm{O}$ site tem o objetivo de divulgar quem é a equipe desenvolvedora, os serviços prestados pelo sistema, formas de entrar em contato com os pesquisadores e link de download do jogo mobile. O site possui uma interface responsiva e adaptável, de acordo com o computador ou dispositivo móvel do usuário.

Ao realizar o login, os professores ou especialistas podem gerenciar as amostras de doenças e pacientes virtuais. Eles podem cadastrar novas doenças e novos sintomas, no módulo. Além disso, eles têm permissão de inserir novas amostras, sendo obrigatório informar os sintomas. Caso a amostra já seja classificada, deve-se inserir a doença. As amostras que não possuem rótulos não servem de entrada/conhecimento para o AM, mas serão classificadas por meio do AM, tomando como base todas as amostras rotuladas. Assim, será possível manter o módulo inteligente sempre atualizado e escalável, pois há possibilidade de crescer, o que é desejável a todo sistema/aplicação. Na Figura 3, pode ser observada a interface das amostras de dados, na visão dos professores.

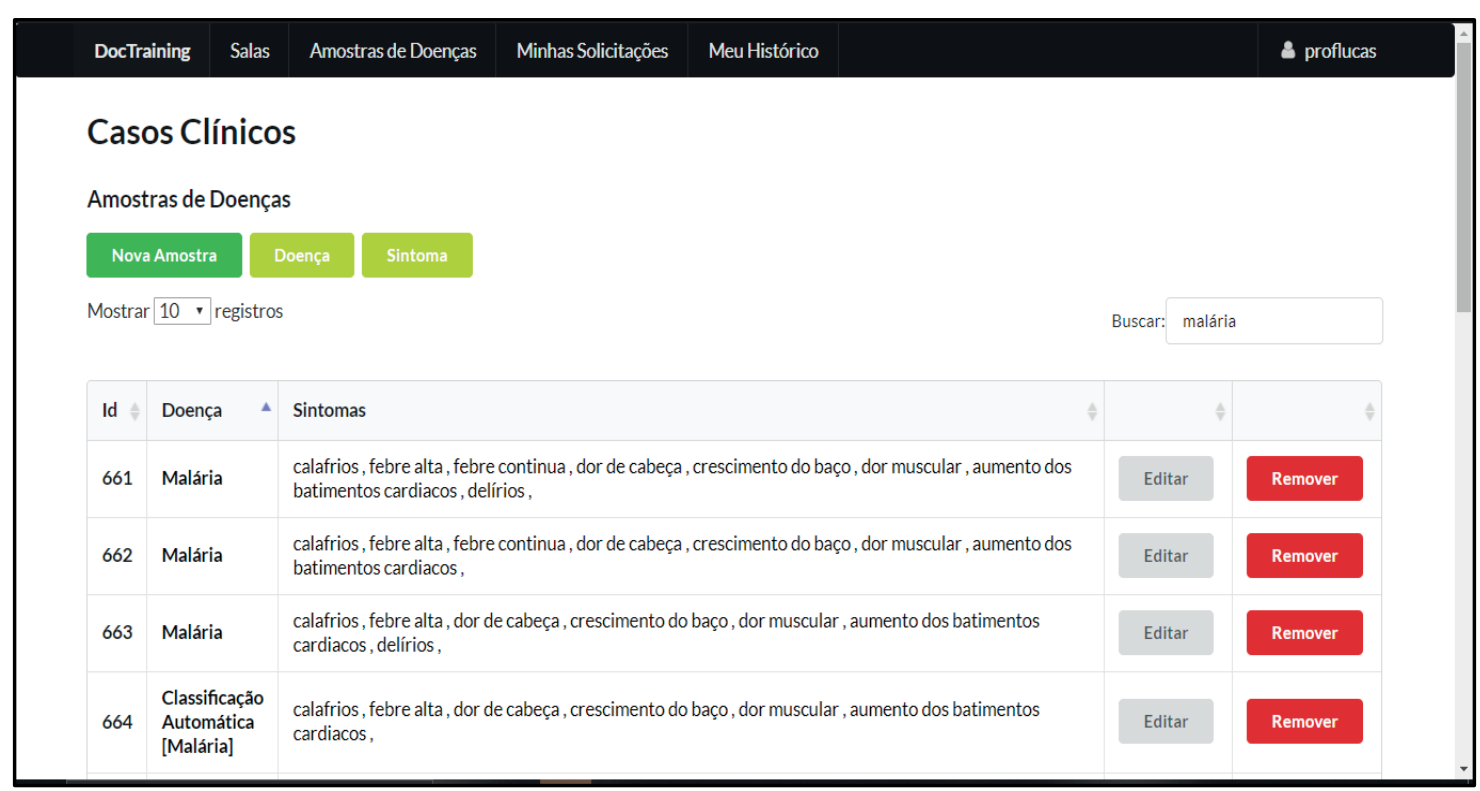

Figura 3 - Tela de gerenciamento de dados do DocTraining.

O sistema conta com dois tipos de visões, sendo uma para administradores, onde é possível visualizar todas as solicitações de alterações das amostras de doenças, todos os logs e os usuários cadastrados no site (podendo desativar o login dos usuários) e a visão dos professores/especialistas, onde não podem ver os usuários cadastrados. As visualizações das solicitações de alterações de amostras de doenças e logs são visíveis quando relacionadas ao professor/especialista que estiver conectado.

Apesar de apenas os professores e especialistas serem capazes de gerenciar os dados, eles não poderão inserir, editar ou deletar os dados diretamente, pois esses dados servem de conhecimento para o AM. Assim, inserir dados repetidos e/ou editar, e/ou apagar informações que se tornem errôneas não se torna proveitoso para aplicação. Desta forma, esses dados antes de serem alterados passam pela verificação e permissão de um dos administradores do DocTraining Mobile, que podem aceitar ou recusar cada uma das solicitações feitas pelos professores ou especialistas. Após avaliação das solicitações, de alterações dos dados, todo histórico dos pedidos é armazenado em uma tabela de log, tornando as informações úteis para auditoria ou relatórios.

${ }^{1}$ http://les.ufersa.edu.br/doctraining 
Também estão disponíveis salas virtuais, como já mencionado anteriormente, na qual o professor consegue adicionar novas salas e novas perguntas em cada uma das salas. Assim, no jogo mobile, os alunos poderão adentrar nas salas, responder as perguntas e adquirir conhecimento de algo específico. Os conteúdos ficam a critério do professor. Os administradores do DocTraining Mobile são capazes de entrar nessas salas, por meio do módulo inteligente, e ver o conteúdo.

\subsection{Web Service do DocTraining Mobile}

Foi desenvolvido um Web Service do DocTraining para realizar a comunicação entres os dois projetos. Esse Web Service foi documentado para facilitar o desenvolvimento do jogo mobile. Assim, quando for desenvolvida uma nova aplicação, será facilitado por conta do Web Service e da documentação disponível.

O Web Service conta atualmente com 10 requisições, sendo elas: todas as doenças, todos os sintomas, todos os casos clínicos, um caso clínico, todos os casos de diabetes, um caso de diabetes, todos os casos de doença cardíaca, um caso de doença cardíaca, todas as salas virtuais e perguntas de uma determinada sala virtual. Todos eles se tratam de métodos GET, ou seja, o jogo mobile faz uma solicitação por meio do método GET e o Web Service retorna os dados correspondentes que estão presentes nas bases de dados e classificados pelo AM. A seguir é ilustrada uma requisição de um caso clínico que, após processado pelo AM, envia as informações para o jogo mobile no formato de JavaScript Object Notation (JSON).

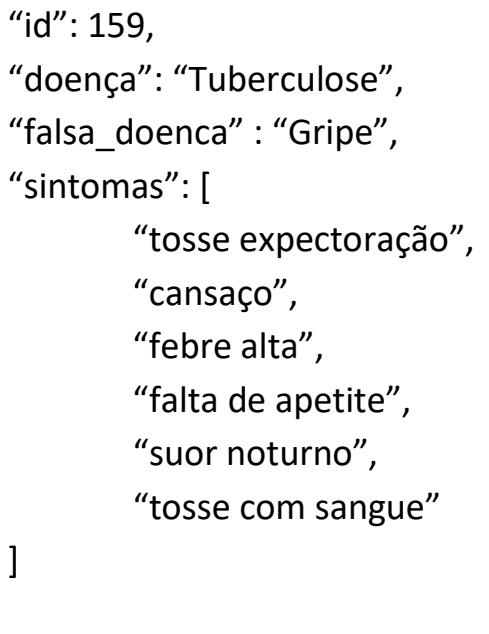

É possível observar que os dados são apresentados mediante a um id, uma doença e uma lista de sintomas. Essa requisição sempre gera uma amostra aleatória, pois no jogo não é interessante apresentar um mesmo problema para o estudante de medicina, mas sim selecionar aleatoriamente, simulando a realidade. O professor pode gerenciar o ambiente de aprendizagem dos seus alunos, assim, as requisições de casos clínicos se tornam interessantes, pois é possível gerenciar o jogo mobile para selecionar quais doenças serão apresentadas aos alunos como motivo de aprendizado.

\subsection{Aprendizado de Máquina}

O AM atua internamente no sistema on-line. A base de dados do hospital possui um modelo de MC para classificar os dados. Ele foi escolhido de acordo com os testes realizados e descritos por Silva (2020), na qual foi desenvolvida e validado a parte inteligente do DocTraining. Essa base possui pacientes virtuais, em que cada paciente possui vários sintomas e uma doença. O modelo é treinado e, em seguida, são feitos testes 
automaticamente, onde os administradores têm acesso a quantidade de acertos e erros que o modelo está tendo nos testes. Em seguida, ele está pronto para realizar a classificação dos dados. O AM classifica também uma doença semelhante com aqueles sintomas, que é usada no jogo para aumentar a complexidade.

Esses dados são rotulados por meio do modelo de $\mathrm{MC}$, formado por Máquina de vetores de suporte, K-Nearest Neighbor, Naive Bayes e Regressão logística, realizando a agregação mediante a Confiança dos classificadores iniciais. Assim, esse modelo de AM gerado pode ser retreinado automaticamente e atualizar a base, caso necessário, pois é possível inserir pacientes virtuais sem que seja especificado a doença dele. A classificação dos dados é feita entre $2 \mathrm{~h}$ e $4 \mathrm{~h}$, e entre $12 \mathrm{~h}$ e 12:30h, sendo horários que, possivelmente, não haverá pico de uso do jogo mobile, que consome o web service, e tão pouco de vários usuários utilizando o sistema on-line.

\section{Avaliação}

Para avaliar a eficácia da solução proposta, foi realizado um experimento quantitativo e qualitativo. A realização do experimento iniciou com uma apresentação do ambiente para os 14 alunos do curso de medicina de uma universidade federal, que contou com o acompanhamento da professora e de um técnico em laboratório. Os alunos utilizaram o jogo durante o período de 17 dias, explorando todas as funcionalidades presentes. Em seguida, foi aplicado um questionário de satisfação para medir o grau de satisfação dos usuários a respeito do jogo e seu uso para educação. O questionário segue o modelo TAM. Vale ressaltar que o sistema on-line e os dados presentes nele foram validados por Silva (2020) através de uma entrevista semiestruturada com esses dois profissionais da medicina citados anteriormente, e obtendo ótimo indício de poder gerenciar e auxiliar ao jogo no ensino-aprendizagem.

O TAM oferece um conjunto de variáveis para explicar a intenção dos usuários em adotar e usar a Tecnologia da Informação, se mostrando como um modelo robusto e confiável. Originado do Theory of Reasoned Action (TRA), o TAM foca na avaliação de duas crenças importantes, que possibilitam explicar a aceitação do usuário e o comportamento de uso, que são: Facilidade de Uso Percebida e Utilidade Percebida (Moura et al. 2014). O questionário foi adaptado para a pesquisa, sendo dividido em três partes: avaliação geral; facilidade de uso e utilidade percebida. Para responder as perguntas, o aluno pode dar uma nota de 1 a 5 , baseadas na escala Likert, sendo que as notas 1, 2, 3, 4 e 5, representam respectivamente, péssimo, ruim, regular, bom e excelente. Esta seção irá abordar as questões de caráter quantitativo.

\subsection{Avaliação Geral}

A primeira parte do questionário de satisfação faz uma avaliação geral do jogo que, em resumo, pergunta se o aluno gostou da experiência em utilizar o DocTraining Mobile e se ele acredita que o jogo pode vir a se tornar um bom meio para estudar ou revisar conteúdos para uma melhor aquisição do conhecimento em momentos livres. A Tabela 1 mostra o resultado das perguntas neste quesito.

Tabela 1 - Pontuações da avaliação geral do jogo.

\begin{tabular}{|c|l|c|c|c|c|c|}
\hline № & Pergunta & \multicolumn{5}{|c|}{ Avaliação } \\
\cline { 3 - 7 } & & Péssimo & Ruim & Regular & Bom & Excelente \\
\hline 1 & $\begin{array}{l}\text { De maneira geral, você gostou da } \\
\text { experiência em utilizar o DocTraining } \\
\text { Mobile? }\end{array}$ & 0 & 0 & 1 & 3 & 10 \\
\hline 2 & $\begin{array}{l}\text { Analisando a proposta do jogo e todo o } \\
\text { seu potencial de crescimento, você } \\
\text { acredita que ele pode vir a se tornar um }\end{array}$ & 0 & 0 & 0 & 1 & 13 \\
\hline
\end{tabular}




\begin{tabular}{|c|l|l|l|l|l|c|}
\hline $\begin{array}{l}\text { bom meio para estudar ou revisar } \\
\text { conteúdos para uma melhor aquisição de } \\
\text { conteúdo em momentos livres? }\end{array}$ & & & & \\
\hline Total & 0 & 0 & 1 & 4 & 23 \\
\hline
\end{tabular}

Com base nos resultados apresentados na Tabela 1, é possível observar que a maioria dos alunos gostaram bastante desta primeira experiência com o jogo. Dos 14 alunos, 10 atribuíram nota máxima na primeira pergunta e 13 deram a mesma nota na segunda. Fica claro que o jogo teve um índice de satisfação relevante, atingindo um total de 23 pontos computados com nota 5 (nota máxima), quando somadas as colunas, o que corresponde a $82,14 \%$ da pontuação total.

\subsection{Facilidade de Uso}

Para garantir uma boa usabilidade ao software, um dos principais pontos observados é a facilidade de uso, ou seja, se a sua interface gráfica é amigável, transmitindo, de forma clara, o objetivo de cada elemento na tela. Por melhor que seja a proposta do software, se a interface que promove a interação do usuário com o sistema não possuir um design de fácil uso, acaba induzindo o usuário a desistir do software, por sentir dificuldades em sua utilização. Dessa forma, quanto mais simples e intuitiva for a interface, mais rápido o usuário irá dominar seu uso.

O design da interface do DocTraining Mobile foi projetado de forma a possuir o mínimo possível de informações na tela, para tentar diminuir o nível de dificuldade inicial, porém, com o passar do tempo, novos recursos serão adicionados ao jogo, que é um processo natural de evolução do software, desde que seja feito de forma planejada. $\mathrm{Na}$ Tabela 2 estão apresentados os resultados obtidos com as questões de satisfação, que diz respeito à Facilidade de Uso do jogo.

Tabela 2 - Pontuações da Facilidade de Uso

\begin{tabular}{|c|c|c|c|c|c|c|}
\hline \multirow[t]{2}{*}{ № } & \multirow[t]{2}{*}{ Pergunta } & \multicolumn{5}{|c|}{ Avaliação } \\
\hline & & Péssimo & Ruim & Regular & Bom & Excelente \\
\hline 3 & $\begin{array}{l}\text { O jogo oferece uma interface de fácil } \\
\text { utilização? }\end{array}$ & 0 & 0 & 0 & 2 & 12 \\
\hline 4 & Foi fácil aprender a jogar o jogo? & 0 & 0 & 2 & 1 & 11 \\
\hline 5 & $\begin{array}{l}\text { Qual nota você daria para a facilidade } \\
\text { de se cadastrar no jogo? }\end{array}$ & 0 & 1 & 0 & 2 & 11 \\
\hline 6 & $\begin{array}{l}\text { De maneira geral, você conseguiu } \\
\text { entender toda a mecânica de } \\
\text { jogabilidade que o jogo oferece? }\end{array}$ & 0 & 0 & 1 & 1 & 12 \\
\hline & Total & 0 & 1 & 3 & 6 & 26 \\
\hline
\end{tabular}

Com base nos resultados da Tabela 2, observa-se que, apesar de alguns alunos sentirem dificuldade em alguns pontos do jogo, como aprender a jogar, se cadastrar e entender a mecânica de jogabilidade, a maioria avaliou o jogo como excelente, em termos de Facilidade de Uso. O uso da interface gráfica do usuário (GUI) foi bem aceita pelos alunos, atingindo um somatório de 46 pontos computados com nota 5 , o que corresponde a $82,14 \%$ da pontuação total, que por coincidência apresentou o mesmo resultado obtido no grau de satisfação.

\subsection{Facilidade de Uso}

Por se tratar de um jogo sério, voltado para a aprendizagem de casos clínicos e outras questões da medicina, é necessário que os jogadores percebam a utilidade do jogo, ou seja, além de se divertirem, eles sintam que estão aprendendo algo novo. Para isso, as perguntas que avaliam este quesito e seus resultados são mostrados na Tabela 3. 
Tabela 3 - Pontuação da utilidade percebida

\begin{tabular}{|c|c|c|c|c|c|c|}
\hline \multirow{2}{*}{ № } & \multirow[t]{2}{*}{ Pergunta } & \multicolumn{5}{|c|}{ Avaliação } \\
\hline & & Péssimo & Ruim & Regular & Bom & Excelente \\
\hline 7 & $\begin{array}{l}\text { O DocTraining Mobile pode ser } \\
\text { utilizado como uma nova forma de } \\
\text { promover o ensino e aprendizagem? }\end{array}$ & 0 & 0 & 0 & 2 & 12 \\
\hline 8 & $\begin{array}{l}\text { O jogo tem potencial de crescimento e } \\
\text { ser utilizado por mais alunos e } \\
\text { professores? }\end{array}$ & 0 & 0 & 0 & 2 & 12 \\
\hline 9 & $\begin{array}{l}\text { Você recomendaria o jogo para um } \\
\text { colega de classe? }\end{array}$ & 0 & 0 & 0 & 2 & 12 \\
\hline & Total & 0 & 0 & 0 & 6 & 36 \\
\hline
\end{tabular}

Os resultados, apresentados na Tabela 3, mostram que os alunos perceberam a utilidade no jogo em termos de aprendizagem, atribuindo notas de bom e excelente. É possível observar que a proposta do jogo, voltada para a educação, ficou clara para os alunos, visto que a atribuição da nota 5 soma um total de 36 pontos, o que corresponde a $85,71 \%$ do total. Esse resultado indica que os alunos acreditam que o jogo pode se tornar uma nova forma de promover o ensino e aprendizagem, já que se trata de um jogo sério e aborda questões práticas da medicina.

\section{Considerações Finais}

Este artigo apresentou os resultados obtidos do DocTraining Mobile, um jogo sério com aprendizado de máquina e um sistema on-line para gerenciar os dados, voltado para o treinamento de estudantes de medicina em casos clínicos, com o intuito de auxiliar o aprendizado dos alunos, simulando pacientes virtuais para serem diagnosticados pelos exames e apresentado diversos outros casos e questões importantes, relacionadas aos casos clínicos. O DocTraining Mobile também conta com salas virtuais que podem ser adicionadas com qualquer conteúdo de medicina para os alunos terem acesso, se baseando no formato de pergunta e resposta de múltipla escolha.

Os professores e profissionais da medicina acessando essa interface podem gerenciar esses dados por meio de um sistema on-line, os dados do hospital são classificados por múltiplos classificadores de aprendizado de máquina, gerando um modelo de classificação que atualiza novos dados inseridos. As informações do sistema on-line são repassadas ao jogo sério por meio de um web service.

Foram aplicados questionários com o público alvo, os estudantes de medicina, para validação. Esses questionários seguiram o método TAM, baseado na escala Likert. Os resultados indicam que os estudantes de medicina acharam fácil o uso do jogo sério e uma boa interface. Além disso, mostrou-se útil, pois é focado na aprendizagem dos próprios estudantes, sendo uma forma de auxiliar no ensino e aprendizagem dos estudantes de medicina, com potencial de crescimento. Estas conclusões se baseiam no questionário TAM, respondido pelos 14 alunos medicina que, de modo geral, gostaram bastante desta primeira experiência com o jogo.

Como trabalhos futuros, é pretendido o planejamento de uma segunda versão do DocTraining Mobile, com base nas melhorias propostas pelos participantes e necessidades dos professores de acordo com o trabalho de Oliveira (2020). Além disso, realizar um estudo experimental com mais turmas e seguindo alguma metodologia de ensino, como por exemplo, Problem Based Learning (PBL) (Boud e Feletti 1998), muito utilizada nas faculdades de medicina (Lopes et al. 2020). E mais, melhorias no web service e no sistema on-line, onde será adicionado um sistema que analisa exames laboratoriais. 


\section{Referências Bibliográficas}

Bogoni, T. N. e Pinho, M. S. Avaliação de um simulador háptico de realidade virtual para treinamento de endodontia. In Brazilian Symposium on Computers in Education (Simpósio Brasileiro de Informática na Educação), 2014 volume 25, page 259.

Boud, D. e Feletti, G. The challenge of problem-based learning. Psychology Press, 1998.

Castro, R. e Siqueira, S. Aprendizagem ativa em sistemas de informação: Novas técnicas propostas e reflexões sobre as experiências. In Anais do XIII Simpósio Brasileiro de Sistemas de Informação, 2017, páginas 535-542. SBC.

Couto, R. C., et al. II anuário da segurança assistencial hospitalar no brasil. Belo Horizonte (BH): Instituto de Estudos de Saúde Suplementar (IESS), 2018.

CREMESP. Exame do cremesp reprova mais da metade dos médicos recém-formados. Acesso em: 23 set. 2017. Disponível em: https://www.cremesp.org.br/pdfs/releasefinal2examecremesp2016.pdf

CREMESP. Exame do cremesp aprova mais da metade dos médicos recém-formados. Acesso em: 15 dez. 2018. Disponível em: https://www.cremesp.org.br/pdfs/RELATORIO EXAME-CREMESP2017.pdf

Davis, F. D. Perceived usefulness, perceived ease of use, and user acceptance of information technology. MIS quarterly, pages 319-340, 1989.

Flores, C. D., et al. Leveraging the learning process in health through clinical cases simulator. In Serious Games and Applications for Health (SeGAH), 2013 IEEE 2nd International Conference on, 2013, páginas 1-6. IEEE.

França, R. M., et al. APS game: jogo sério de simulação de casos clínicos aplicado a educação continuada em saúde no Brasil. Jornal Brasileiro de TeleSSaúde, 2016.

Fu, P., Peng, Q., e Hu, X. A web service composition system based on semantic parsing. In 2015 IEEE 19th CSCWD, 2015, pages 561-569. IEEE.

James, J. T.. A new, evidence-based estimate of patient harms associated with hospital care. Journal of patient safety, 9(3):122-128, 2013.

Lopes, G. Q. Vinte erros comuns na prática clínica. Acesso em: 10 set. 2020. Disponível em: https://pebmed.com.br/vinte-erros-comuns-na-pratica-clinica/

Lopes, R. M., Hauser-Davis, R. A., Oliveira, M. M., Pierini, e Outros. Principles of problem-based learning for training and professional practice in ecotoxicology. Science of The Total Environment, v. 702, p. 134809, 2020.

Moura, F. L., Ferreira, F. A., e Barros, V. F. d. A. Aplicação do modelo de aceitação de tecnologia para avaliar a aceitação e uso de software ERP. In INTERTECH, 2014.

Oliveira, E. J. DocTraining Mobile: Um jogo sério para treinamento de estudantes de medicina em casos clínicos. Mossoró: UFERSA/UERN, 2020. 104p. Dissertação de Mestrado.

Orton, E. e Mulhausen, P. E-learning virtual patients for geriatric education. Gerontology \& geriatrics education, 28(3):73-88, 2008.

Silva, J. C. P. Um módulo inteligente baseado em aprendizado de máquina para treinamento de estudantes de medicina no DocTraining. Mossoró: UFERSA/UERN, 2020. 111p. Dissertação de Mestrado.

Silva, J. C. P., de Oliveira, E. J., Neto, F. M. M., de M Santos, A., Queiroz, P. G. G., \& de Freitas, A. A. C. Aprendizado de máquina nos jogos para medicina: Uma revisão sistemática. In: Anais do XIX Simpósio Brasileiro de Computação Aplicada à Saúde. Porto Alegre, RS, Brasil: SBC, 2019. páginas 70-81. 